\title{
Banding of oesophageal varices using locally improvised materials
}

\author{
ES Wijewickrama ${ }^{1}$, IWMP Wanigasuriya ${ }^{2}$ and SD Jayaratne ${ }^{3}$
}

\begin{abstract}
Bleeding from oesophageal varices is the commonest cause of significant upper gastrointestinal bleeding in Sri Lanka. Endoscopic band ligation is an effective method in the management of varices. But the cost of banding equipment is high, unaffordable for a majority of our patients. We have devised a cheap method to carry out banding of varices.

Banding of varices using this technique was carried out in 235 patients. In the patients who were followed up, there was a reduction in the size of varices across all grades of varices. No complications due to banding were noted, and only 10 patients developed re-bleeding. Since the initial experience on efficacy and safety of this technique is encouraging, we believe that its widespread adoption in Sri Lanka would be cost effective and lifesaving.
\end{abstract}

Bleeding from oesophageal varices is the commonest cause of significant upper gastrointestinal bleeding in Sri Lanka and portal hypertension due to alcohol induced cirrhosis is the commonest cause of varices worldwide. Mortality from first bleed is about 50\% [1]. Patients who survive the first bleed are at high risk of re-bleeding (over $60 \%$ at 1 year) [2,3]. The risk of bleeding increases with the severity of liver disease and variceal size [4]. Endoscopic band ligation is an effective method in arresting acute bleeding and in preventing re-bleeding from oesophageal varices $[5,6]$. It is also considered to be safer and more effective than endoscopic injection sclerotherapy [2,6-9].

The cost of banding is high in Sri Lanka. The price of a single commercially available set of bands range from Rs 15000 to Rs 20000 . Thus banding is unavailable for a majority of the patients with variceal bleeding despite the availability of endoscopic facilities in many hospitals in Sri Lanka. We have devised a cheap method from locally available materials for banding of varices.

14CH/Fr Foley catheters were used to make 1/16 inch thick bands. Up to 150 bands could be made from a single catheter. Thread seal was used to fasten the bands to a plastic cylinder at the end of the endoscope. The same technique, using the handle unit and the ligating unit of the commercially available kit, was followed in the insertion of the bands. The cost of a single application of bands was about Rs 5.

This technique was followed at the endoscopy unit of Colombo South Teaching Hospital in banding of oesophageal varices since March 2000. Banding was performed in patients with acute variceal bleeding as well as in those who were detected as having varices on upper GI endoscopy. Informed verbal consent for the procedure was obtained from the patients whenever possible. Paguet's endoscopic grading of varices was used to grade the size of oesophageal varices [10].

A total of 235 patients were banded using this method between March 2000 and January 2004. A majority of these patients (about 90\%) underwent banding immediately following acute upper gastrointestinal bleeding: six patients died during the first week. All the patients who died were very ill and had already lost a large amount of blood by the time they were subjected to banding.

Eighty eight (38.4\%) patients attended at least one follow up endoscopy session. These patients were followed up for variable periods of time ranging from 1 to 30 months. Fifty two patients $(59.1 \%)$ were followed up for 0 to 6 months while 25 (28.4\%) were followed up for 6 to 12 months, and the rest up to 30 months.

Patients' ages ranged from 7 to 79 with a median of 52. Eighty three per cent were males. A total of 198 banding sessions were carried out and 777 bands were inserted with an average of four bands per session.

Most of the patients showed a reduction in the size of oesophageal varices during the follow up period (Table 1). Only five (5.7\%) patients showed worsening of varices. None had complications such as ulceration or secondary bleeding in the immediate post-procedure period. Only $10(11.4 \%)$ patients developed re-bleeding during the follow up period.

Our study shows that endoscopic band ligation can be carried out in patients with oesophageal varices using our locally improvised bands. This has an enormous impact on the cost of banding making it an affordable option for patients with varices admitted to government hospitals.

Table 1. Severity of oesophageal varices at the initial banding and on follow up endoscopy

\begin{tabular}{lccrrrr}
\hline Severity of varices & At initial banding & & \multicolumn{4}{c}{ At follow up endoscopy } \\
& & None & Grade I & Grade II & Grade III & Grade IV \\
\hline Grade I & - & & & & & \\
Grade II & 19 & - & 11 & 4 & 4 & - \\
Grade III & 54 & 3 & 21 & 17 & 12 & 1 \\
Grade IV & 15 & - & 3 & 6 & 3 & 3 \\
Total & $\mathbf{8 8}$ & $\mathbf{3}$ & $\mathbf{3 5}$ & $\mathbf{2 7}$ & $\mathbf{1 9}$ & $\mathbf{4}$ \\
\hline
\end{tabular}




\section{Brief reports}

The poor follow up rate was a deficiency in our study. This was not unexpected considering that a majority were heavy alcohol consumers who had a low educational level and belonged to a poor socioeconomic class. The extremely low re-bleeding rate observed could be due to a higher abstinence rate and better compliance with treatment in the group who attended followed up. Despite the poor follow up rate, almost all showed a reduction in the size of oesophageal varices, with no complications across all grades of varices. A study which compares the use of these bands against the use of standard bands with a more complete follow up would have strengthened the efficacy of our technique. We have already made plans for such a study. Nevertheless, the results of the initial experience are encouraging. Hence we believe that widespread adoption of this technique in Sri Lanka would be cost effective and life-saving.

\section{References}

1. Christensen E, Fauerholdt L, Schlichting P, Juhl E, Poulsen $\mathrm{H}$, et al. Aspects of the natural history of gastrointestinal bleeding in cirrhosis and the effect of prednisone. Gastroenterology 1981; 81: 944-52.

2. D'Amico G, Pagliaro L, Bosch J. The treatment of portal hypertension: a meta-analytic review. Hepatology 1995; 22: 332-54.

3. de Franchis R. Updating consensus in portal hypertension: Report of the Baveno III Consensus Workshop on def- initions, methodology and therapeutic strategies in portal hypertension. Journal of Hepatology 2000; 33: 846-52.

4. Prediction of the first variceal haemorrhage in patients with cirrhosis of the liver and oesophageal varices. A prospective multicentre study. The North Italian Endoscopic Club for the Study and Treatment of Oesophageal Varices. New England Journal of Medicine 1988; 319: 983-9.

5. Bosch J, Garcia-Pagan JC. Complications of cirrhosis I: Portal hypertension. Journal of Hepatology 2000; 32: 141-56.

6. Gimson AES, Ramage JK, Panos MZ, Hayllar K, Harrison $\mathrm{PM}$, et al. Randomised trial of variceal banding versus injection sclerotherapy for bleeding oesophageal varices. Lancet $1993 ; \mathbf{3 4 2}$ : 391-4.

7. Burroughs AK, Vangeli M. Transjugular intrahepatic portosystemic shunt versus endoscopic therapy: randomized trials for secondary prophylaxis of variceal bleeding: an updated meta-analysis. Scandinavian Journal of Gastroenterology 2002; 37: 249-52.

8. de Franchis R, Primigani M. Endoscopic treatment for portal hypertension. Seminar of Liver Disease 1999; 19: 439-55.

9. Groszmann RJ, Garcia-Tsao G. Endoscopic variceal banding vs. pharmacological therapy for the prevention of recurrent variceal haemorrhage: what makes the difference? Gastroenterology 2002; 123: 1388-91.

10. Paguet J. Prophylactic sclerosing treatment of the oesophageal wall in varices: A prospective controlled study. Endoscopy 1982; 14: 4.

${ }^{1}$ Intern Medical Officer and ${ }^{2}$ Nursing Officer, Endoscopy Unit, Colombo South Teaching Hospital, ${ }^{3}$ Senior Lecturer in Medicine, Faculty of Medical Sciences, University of Sri Jayawaredenepura, Nugegoda, Sri Lanka.

Correspondence: ESW, Tel.: 2517853,0777-270624, e-mail: <erangasw@yahoo.com>. 